\title{
EXPERIMENTAL STUDY OF THE ELECTRIC WIND INDUCED BY A DIELECTRIC BARRIER DISCHARGE PLASMA ACTUATOR
}

\author{
${ }^{* 1}$ Julie Vernet, ${ }^{* 1}$ Ramis Örlü, ${ }^{* 2}$ Gunilla Efraimsson, ${ }^{* 1} \mathrm{P}$. Henrik Alfredsson \\ ${ }^{* 1}$ Linné Flow Centre, KTH Mechanics, Royal Institute of Technology, S-100 44 Stockholm, Sweden \\ ${ }^{* 2}$ Linné Flow Centre, KTH Aeronautical and Vehicle Engineering, Royal Institute of Technology, S-100 44 Stockholm, Sweden \\ julie@mech.kth.se, ramis@mech.kth.se, gef@kth.se, hal@mech.kth.se
}

\begin{abstract}
An experimental study is conducted on the electric wind produced by Dielectric Barrier Discharge (DBD) plasma actuators placed at the top of a half cylinder. Parameters as driving voltage, frequency, dielectric thickness and their influence on the efficiency of the actuator are investigated by means of velocity measurements using Laser Doppler Velocimetry. Mean profiles show that the plasma actuators are able to produce wall jets with velocities up to $4.5 \mathrm{~m} / \mathrm{s}$. Increasing the driving voltage and frequency increases the jet velocity and thus the momentum added by the actuators. The time variation of the electric wind is also investigated through phaseaveraged measurements. The increase of induced velocities during both the forward and backward stroke of the high-voltage cycle shows agreement with the PUSH-push mechanism theory of DBD plasma actuators.
\end{abstract}

\section{INTRODUCTION}

The first demonstration of Dielectric Barrier Discharge (DBD) plasma actuators for aerodynamic purposes was done by Roth's group (see e.g. [1]) and since then plasma actuators have shown interesting abilities for active flow control [2,3]. DBD plasma actuators make flow control possible by adding momentum to the surrounding air close to the actuator surface during the ionization process. Drag reduction and lift enhancement by separation control [4-9], transition delay [10,11] and turbulent boundary layer control [12] are being investigated both experimentally and numerically by various research groups.

In order to use DBD plasma actuators in an efficient way, knowledge of the flow induced by the plasma, also called electric wind, is necessary. Research groups studied this phenomenon of momentum addition and its spatial distribution using non-intrusive experimental methods such as Particle Image Velocimetry (PIV) and Laser Doppler Velocimetry (LDV). Kriegseis et al. [13] review in their paper different methods to estimate the force induced by the plasma actuator on its surrounding using such velocity measurements. Fortes et al. [14] made a study of the dependence of the induced jet velocities on the geometry of the actuator and parameters of the driv- ing current of the power supply. In Ref. [15], Erfani et al. test different configurations of embedded electrodes and record the induced velocity magnitudes. The impact of atmospheric temperature and pressure changes on the plasma actuator performances has been investigated by Versailles et al. [16], whereas Erfani et al. [17] study the influence of the dielectric temperature on the induced flow field. Ranges of velocities and thus forces as well as spatial developments of the jet differ from one study to another due to differences in geometry, driving current parameters and material of the actuators used by the different research groups.

In the present study, we start by investigating the mean wall-normal velocity profiles induced by in-house built DBD plasma actuators and investigate the electric wind dependence on the driving voltage, frequency and dielectric thickness as well as its streamwise development. The present mean flow data will also serve as calibration data for a numerical model of the actuator force used in a companion project [18].

Since an alternating high-voltage power supply is necessary to sustain the discharge of the DBD plasma actuators, one may think about the electric wind as an unsteady and periodic flow. Numerical studies have shown voltage-cycle dependence and asymmetric development of the induced airflow during its forward and backward strokes $[19,20]$. The forward stroke corresponds to the negative half-cycle of the alternating driving voltage, meaning that the exposed electrode is negatively charged, when the backward stroke is the positive half-cycle. However, only few experimental studies have investigated this phenomenon and among those, two different schools appeared. Enloe et al. [21] use Corke's definition [3] to qualify their induced pulsed wall-jet of PUSH-push mechanism, meaning that both strokes of the driving voltage produce a force directed from the actuator to the surrounding with a clear dominance of the force applied during the forward stroke of the driving voltage. Time-resolved measurements of Forte et al. [14] seem to indicate the same result. However, Neumann et al. [22] claim having plasma actuators producing discharge with a PUSH-pull mechanism, meaning that it is only during the forward stroke that the force is in the streamwise direction. Debien et al. [23] compared 

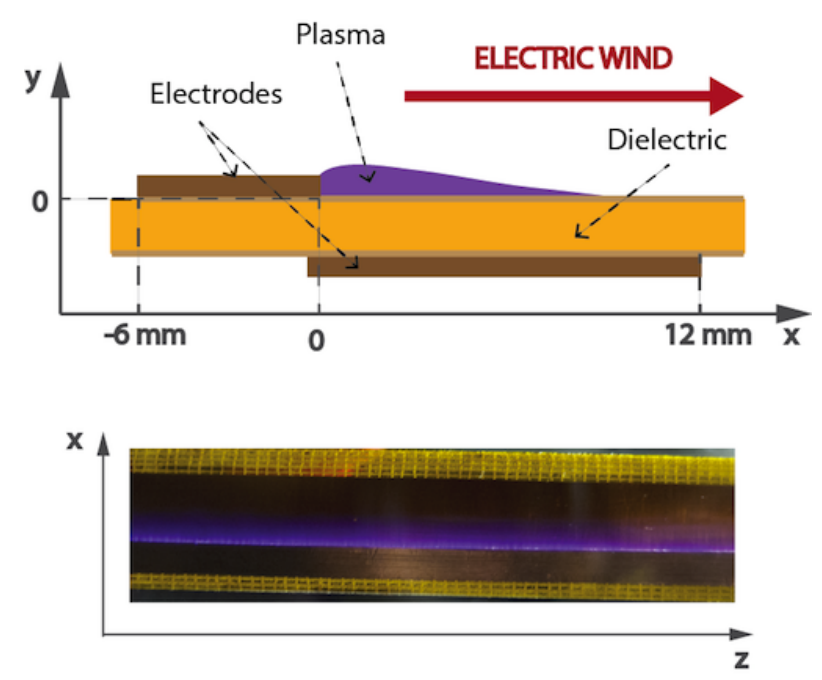

Fig. 1 Sketch of the actuator and coordinate system description. The bottom picture is a top view of the glowing discharge.

DBD plasma actuators built with different electrode geometries and conclude that the electro-hydro-dynamic (EHD) force produced by the plasma is dependent on the top electrode shape. Kotsonis and Ghaemi [24] showed that different voltage waveforms, at a fixed driving frequency, can induce either PUSH-push or PUSH-pull behaviour of their actuator. In our study, we underline this phenomenon by studying phase-averaged LDV measurement data and try to further understand the dependency of these mechanisms on the various involved parameters.

\section{EXPERIMENTAL SET UP}

\subsection{DBD plasma actuators and power supply}

DBD plasma actuators are constructed from two electrodes separated by a dielectric material, see Fig. 1 and e.g. Cristofolini et al. [25]. In the present study we use $66 \mu \mathrm{m}$ thick adhesive copper foil for the two $14 \mathrm{~cm}$ long rectangular electrodes disposed asymmetrically above and below a dielectric material. In order to ensure a homogeneous plasma generation in the spanwise direction, the electrodes are overlapping with about $1 \mathrm{~mm}$. The electrode on the top of the dielectric material is $6 \mathrm{~mm}$ long in the streamwise direction whereas the bottom electrode is $13 \mathrm{~mm}$, in order not to limit the streamwise length of the plasma. The dielectric sheet of the actuator is made of two layers of adhesive Kapton, $70 \mu \mathrm{m}$ thick each, separated by a sheet of Mylar as suggested to us by Andrea Cristofolini (personal communication). Two types of Mylar with different thicknesses have been used in this study; the actuator named Act. 1 is built with $0.25 \mathrm{~mm}$ thick Mylar but for the other actuator, Act. 2, the Mylar sheet is $0.35 \mathrm{~mm}$ thick. The dielectric of Act. 1 is thus $0.39 \mathrm{~mm}$ thick $\left(\delta_{1}\right)$ resulting in a total thickness for the actuator of $0.52 \mathrm{~mm}$ whereras the dielectric of Act. 2 is $0.49 \mathrm{~mm}$ thick $\left(\delta_{2}\right)$ and the to-

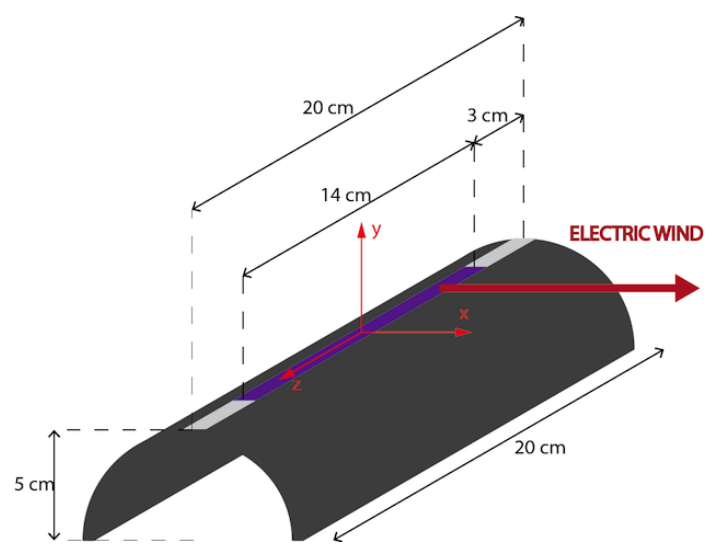

Fig. 2 Plasma actuator mounted at the top of the halfcylinder

tal thickness of the actuator is $0.62 \mathrm{~mm}$. The spanwise length of the dielectric is $6 \mathrm{~cm}$ longer than the actuation length, with $3 \mathrm{~cm}$ on each side as shown in figure 2, to avoid spark generation between the electrodes on the sides of the actuator and ensure operator safety.

To produce the high-voltage alternating current needed to sustain the discharge produced by the DBD plasma actuators we use a combination of a standard sinusoidal signal generator and a high voltage amplifier. The gain of the amplifier is 2000 and it can output peakto-peak voltages up to $20 \mathrm{kV}_{p-p}$. However, the slew rate of the present amplifier limits the driving frequencies to values lower or equal to $2 \mathrm{kHz}$. Finally, the amplified high-voltage output is linked to the top electrode of the plasma actuator whereas the bottom electrode is grounded.

\subsection{LDV measurements set-up}

In this paper, the electric wind induced by actuators placed on the top of a half-cylinder made of Plexiglas was studied, see Fig. 2. The top electrode is thus exposed to the surrounding air whereas the bottom electrode is embedded between the dielectric and the cylinder surface. This geometry was chosen since it is relevant for future planned separation control experiments. The cylinder is placed inside a Plexiglas box in order to reduce disturbances from the surroundings on the electric wind.

Laser Doppler Velocimetry has been employed to record the velocity of the airflow using a singlecomponent Dantec Dynamic LDV FlowLite system with a BSA 60 processor. The measurement volume is created by the intersection of two $632.8 \mu \mathrm{m}$ wavelength laser beams. The focal length of the laser optics is $160 \mathrm{~mm}$ and the part of the box separating the laser head from the plasma actuator was built in glass in order to minimize optical distortions. The box containing the cylinder was mounted over a manual traversing system with a precision of $0.05 \mathrm{~mm}$ to be able to translate it in the streamwise direction. The laser head was attached to the vertical axis of an ISEL C142-4 traversing system that could be controlled and synchronized with the mea- 


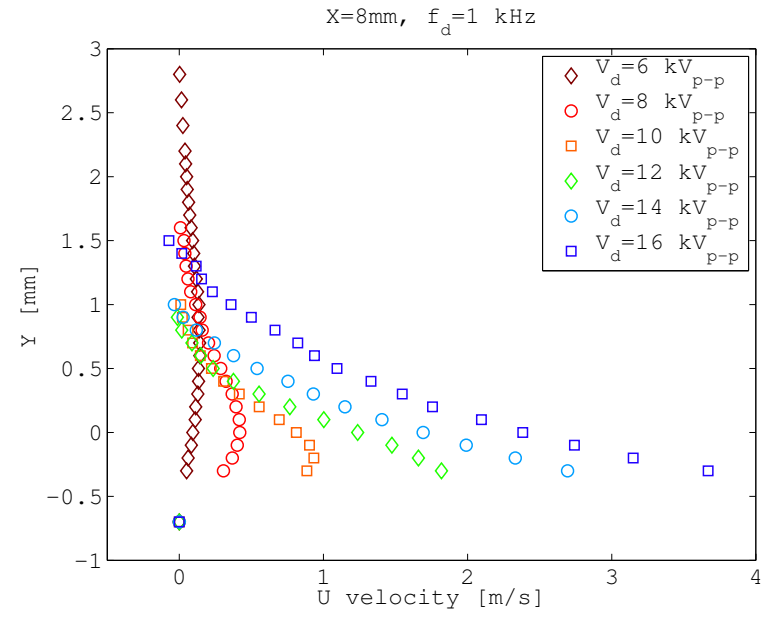

Fig. 3 Evolution of the mean wall-normal velocity profiles as a function of the driving voltage. Wall position is at $\mathrm{Y}=-0.66 \mathrm{~mm}$.

surement software of the BSA system. DEHS droplets with a diameter of about $1 \mu \mathrm{m}$ were used to seed the induced flow and 50000 samples were recorded for each measurement point at a sample rate of about 2000 particles/sec. The transit-time weighting method has been used to evaluate statistical quantities.

\section{ELECTRIC WIND STUDY AND DESCRIPTION}

\subsection{Parametric study of the induced mean flow field}

Before using plasma actuators for separation control purpose, it is essential to have knowledge of the induced flow field which is directly linked to the force produced by the actuator. This investigation will also provide a data base for the calibration and comparison with the numerical model [18] of the actuation force.

The influence of three parameters known to have a strong affect on the electric wind is investigated:

- The driving voltage applied between the two electrodes $\left(4<V_{d}<18 \mathrm{kV}_{p-p}\right)$

- The driving frequency $\left(0.5<f_{d}<2 \mathrm{kHz}\right)$

- The thickness of the dielectric $\left(\delta_{1}=0.39 \mathrm{~mm}\right.$ and $\left.\delta_{2}=0.49 \mathrm{~mm}\right)$

The mean streamwise component $(U)$ of the wallnormal (or rather vertical) velocity profile for the different driving voltages can be observed in Fig. 3. In this figure, the profiles were recorded $8 \mathrm{~mm}$ downstream the edge of the exposed electrode and with a driving frequency of $1 \mathrm{kHz}$. For $V_{d}=4 \mathrm{kV}_{p-p}$, no discharge could be observed on the surface of the actuator and the LDV measurements revealed a zero-velocity profile, meaning that the driving voltage was too low for the discharge to initiate. However, for $V_{d}=6 \mathrm{kV}_{p-p}$, small, but non-zero

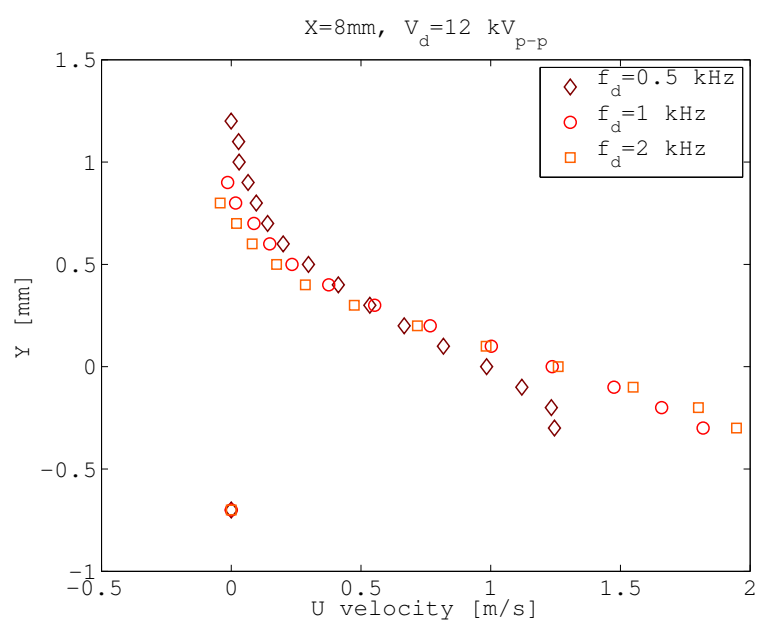

Fig. 4 Evolution of the mean wall-normal velocity profiles as a function of the driving frequency. Wall position is at $\mathrm{Y}=-0.66 \mathrm{~mm}$.

velocities could be observed indicating that the breakdown voltage [3] was contained between 4 and $6 \mathrm{kV}_{p-p}$. As shown previously in Ref. [14], the flow fields induced by the different voltages are similar to wall-jet profiles and the velocities of the jets increase with the driving voltage with values from $0.13 \mathrm{~m} / \mathrm{s}$ to $3.67 \mathrm{~m} / \mathrm{s}$ for our case. However when the driving voltage increased to $18 \mathrm{kV}_{p-p}$, the discharge became unstable, plasma filaments and sparks were visible and after a few minutes started to damage the actuator. Figure 3 also shows that the extremity of the jet moves closer to the surface of the cylinder (which is at $\mathrm{Y}=-0.66 \mathrm{~mm}$ for $\mathrm{X}=8 \mathrm{~mm}$ ) when the voltage increases. The maximum velocities of the jet were not recorded for the cases of $V_{d}=14$ and $16 \mathrm{kV}_{p-p}$ since they are probably below the lowest point where we were able to measure. This last result would imply that velocities even higher than $3.67 \mathrm{~m} / \mathrm{s}$ can be induced by the actuator at the streamwise position of $\mathrm{X}=8 \mathrm{~mm}$.

Similar results were obtained when increasing the driving frequency at constant driving voltage; it increased the induced jet velocities, see Fig. 4. The increase of $f_{d}$ from 0.5 to $1 \mathrm{kHz}$ seems to have a larger effect than the increase from 1 to $2 \mathrm{kHz}$. However this could be due to the slew rate of the amplifier and thus its abilities to follow the voltage variations for high frequencies. The frequency dependence study will be improved by further measurements using a different power supply able to work in the frequency range of 5 to $20 \mathrm{kHz}$ in the near future.

Comparison of results obtained for the two different dielectric thicknesses using driving voltages of 6,12 and $16 \mathrm{kV}_{p-p}$ are shown in Fig. 5. The wall position was different for the two actuators since the Act. 2 is $0.1 \mathrm{~mm}$ thicker than Act. 1. According to the literature [14], thicker dielectric layers usually allow the use of higher voltages thereby inducing stronger wall jets. However, in our case the discharge of Act. 2 also became unstable for $V_{d}=18 \mathrm{kV}_{p-p}$, limiting the increase of the driving 


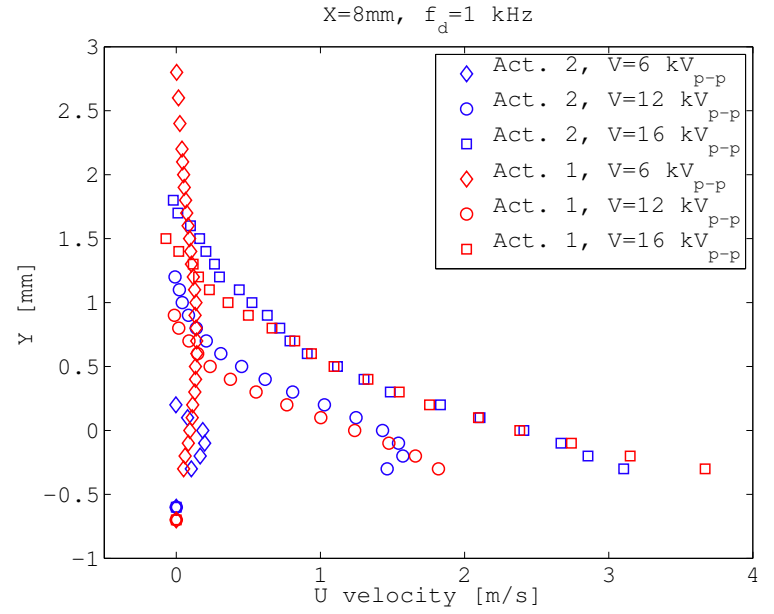

Fig. 5 Mean wall normal velocity profiles for Act. 1 and Act. $2\left(\delta_{1}=0.39 \mathrm{~mm}, \delta_{2}=0.49 \mathrm{~mm}\right)$ at different driving voltages. Wall positions are at $\mathrm{Y}_{1}=-0.66 \mathrm{~mm}$ for Act. 1 and $\mathrm{Y}_{2}=-0.56 \mathrm{~mm}$ for Act. 2.

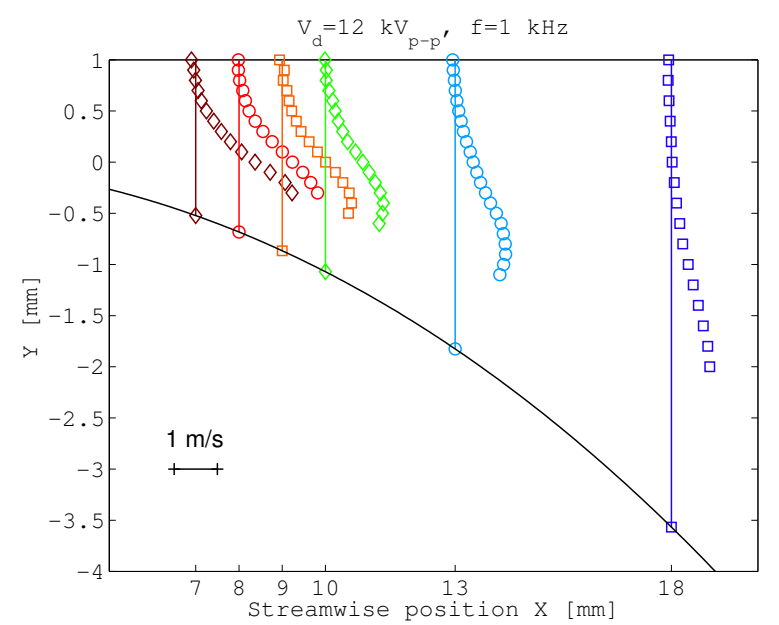

Fig. 6 Streamwise evolution of the mean wall-normal velocity profiles. The cylinder wall is represented by the black line.

voltage. Looking at the induced flow field one can notice that slightly lower velocities are induced when using Act. 1 for the driving voltage $6 \mathrm{kV}_{p-p}$ but for $V_{d}=12$ and $16 \mathrm{kV}_{p-p}$, the reverse conclusion can be drawn. These results are in agreement with the observations in Ref. [14]. Since Act. 1 was able to produce stronger wall jets for most of the tested driving voltages, it has been selected as the one that will be used for future separation control experiments.

In figure 6 , the streamwise development along the cylinder is shown. Unfortunately the present set up did not allow measurement close to the cylinder surface but the results still show that the flow follows the cylinder surface. This is one of the advantages with the flexibility of the actuators, namely that they adopt the shape of the surface and can thus produce a jet that is oriented tangentially to the surface. In a numerical companion project this type of results will be used to verify the

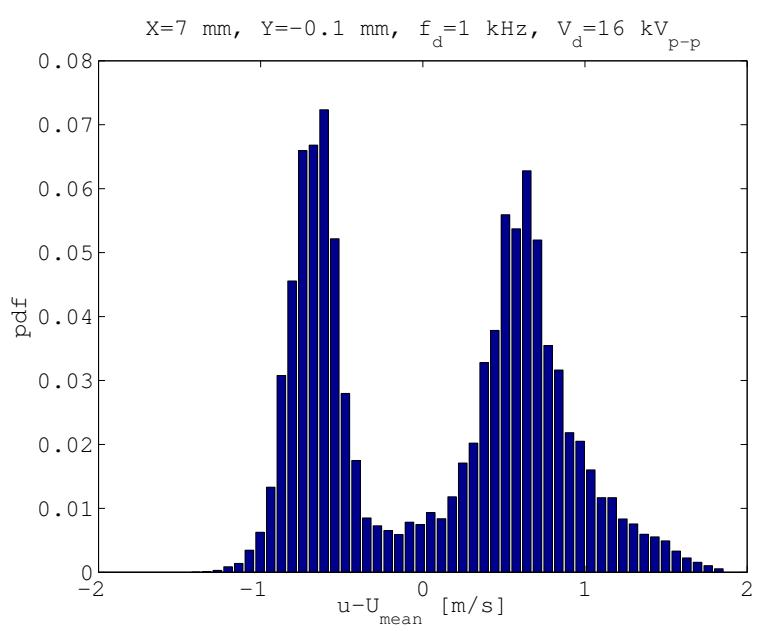

Fig. 7 Bi-modal pdf representative of two ranges of velocities, one for each stroke of the high-voltage cycle. $\mathrm{U}_{\text {mean }}=2.84 \mathrm{~m} / \mathrm{s}$.

model used for the actuation force.

\subsection{Phase-averaged results}

The probability density function (pdf) of the velocity close to the actuator shows a bi-modal character as shown in Fig. 7. In the figure, the mean value of the velocity $(2.84 \mathrm{~m} / \mathrm{s}$ at this position) has been subtracted. This shows that the velocity always is in the positive $\mathrm{X}$-direction. The bi-modal behaviour is coupled to the forward and backward strokes of the driving signal. In order to further study this phenomenon, the time signal was phase averaged over one period of the driving signal giving the results shown in Fig. 8. The period was divided into 200 bins over which the Chauvenet's criterion was applied, see Coleman and Steele [26], p.48, and the weighting average was done. The figure shows both the phase averaged variations and all the remaining individual samples giving an indication of the spread. One can see how the signal is divided into two different segments, a large increase in velocity is observed during the first half of the period (forward stroke), whereas after the decay a second smaller increase is observed (backward stroke). Although we expect the electric wind to be laminar, the spread around the phase averaged mean value could indicate some cycle-to-cycle variation of the electric wind generation.

Figure 9 shows the acceleration derived from the phase-averaged velocity. As apparent (which is also obvious from Fig. 8) the induced flow shows positive acceleration twice during a period, with the first (in the figure) acceleration being much stronger than the second. This clearly indicates a PUSH-push behaviour of the present set up.

The variation of the pdf at a specific position for different driving voltages is illustrated in Fig. 10. It is shown that the development length of the wall jet changes with the strength of the driving voltage, a low voltage will result in a shorter development length and 


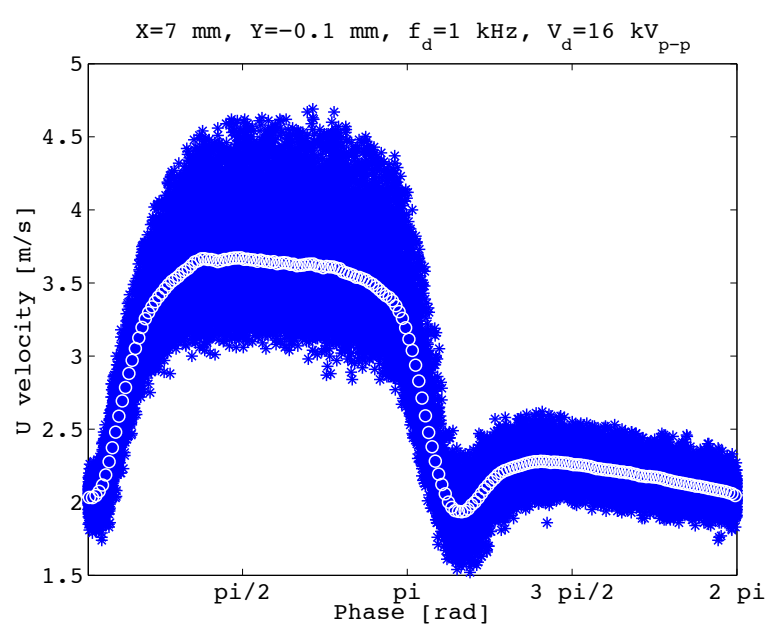

Fig. 8 Complete velocity time-series in blue and phase-averaged velocity in white.

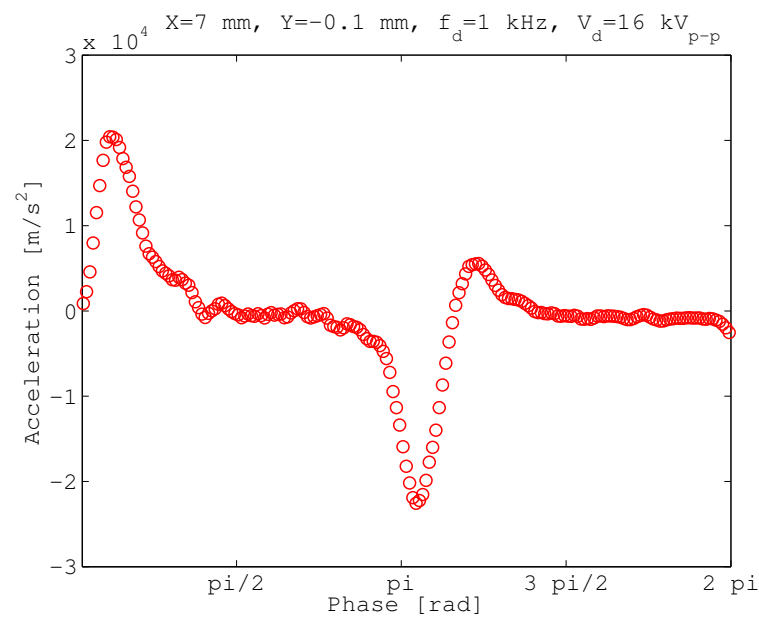

Fig. 9 Flow acceleration obtained from the phase-averaged velocity signal.

a smaller variation around the mean. However, also the mean velocity decreases with decreasing strength of the voltage. Figure 11 on the other hand shows the streamwise development of the pdf for two different driving voltages. It is clearly seen that for the higher voltage the bimodal distribution is sustained further downstream than for the lower voltage.

\section{SUMMARY AND CONCLUSIONS}

In this work a DBD plasma actuator has been developed and the induced airflow characterized through LDV measurements. The results and conclusions can be summarized as follows:

- Jet velocities increase with driving voltage and frequency. The extremity of the wall jet (maximum velocity) moves closer to the wall when the driving voltage increases.

- The thinner dielectric used produces higher jet velocities for almost all the voltages of interest.

- The jet is found to follow the surface of the cylinder.
- The bi-modal behavior of the pdf as well as the phase-averaged measurements are in accordance with the two strokes (forward and backward) of the voltage cycle. This shows that for the present configuration the actuator behaves according to the PUSH-push mechanism.

The present actuator setup will be used to control separation in a bluff body experiment, and to develop a numerical model of the force produced by the actuators for implementation in numerical control studies.
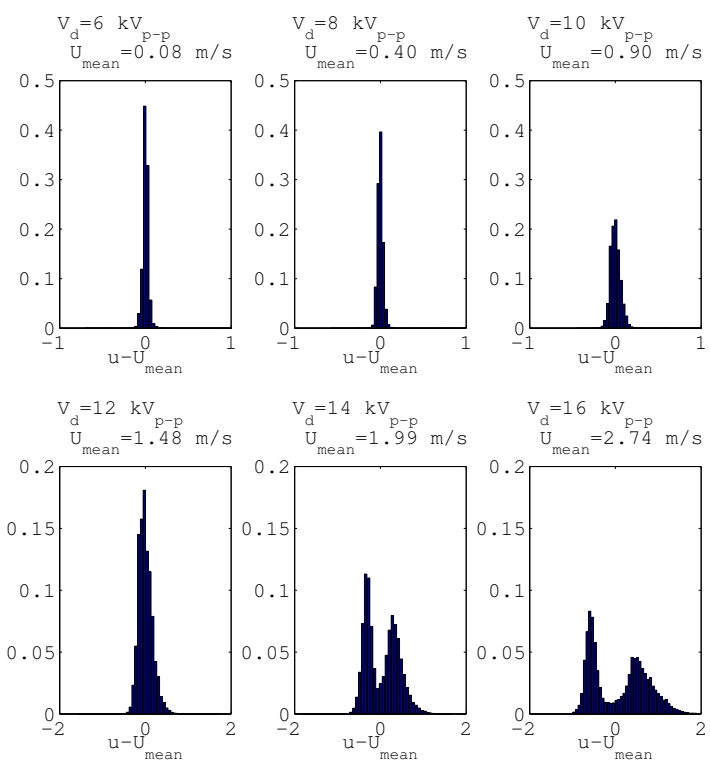

Fig. 10 Pdf of velocities for different driving voltages at the driving frequency $f_{d}=1 \mathrm{kHz}$ and position $\mathrm{X}=8 \mathrm{~mm}$ and $\mathrm{Y}=-0.1 \mathrm{~mm}$.

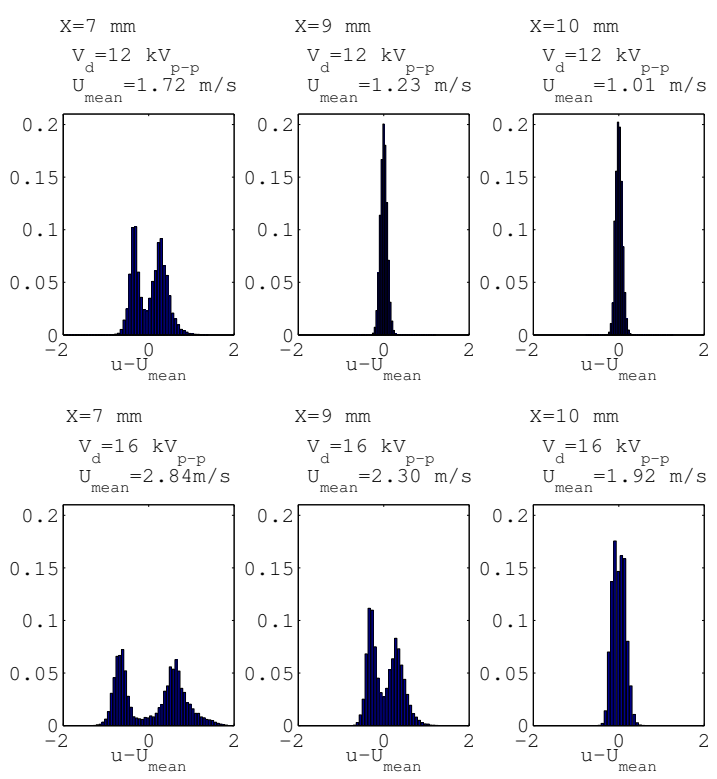

Fig. 11 Pdf of velocity at three downstream positions $(\mathrm{X}=7,8,9 \mathrm{~mm}), \mathrm{Y}=-0.1 \mathrm{~mm}$, for two different driving voltages. Upper row $12 \mathrm{kV}$, lower row $16 \mathrm{kV}$. 


\section{REFERENCES}

[1] Roth, J. R., Sherman, D., M., Electrohydrodynamic Flow Control with a Glow-Discharge Surface Plasma, AIAA J., 38-7, pp. 1166-1172 (2000).

[2] Moreau, E., Airflow Control by Non-Thermal Plasma Actuators, J. Phys. D: Appl. Phys., 40, pp. 605-636 (2007).

[3] Corke, T. C., Enloe, C. L., Wilkinson, S. P., Dielectric Barrier Discharge Plasma Actuators for Flow Control, Annu. Rev. Fluid Mech., 42, pp. 505-529 (2010).

[4] Post, M. L., Corke, T. C., Separation Control on High Angle of Attack Airfoil Using Plasma Actuators, AIAA J., 42-11, pp. 2177-2184 (2004)

[5] Huang, J., Corke, T. C., Thomas, F. O., Plasma Actuators for Separation Control of Low-Pressure Turbine Blades, AIAA J., 44-1, pp. 51-57 (2006)

[6] Abdoli, A., Mirzaee, I., Anvari, A., Purmahmod, N., Simulation of Body Force Field Effects on Airfoil Separation Control and Optimization of Plasma Actuator, J. Phys. D: Appl. Phys., 41, 175204 (2008)

[7] Jukes, T. N., Choi, K.-S., Control of Unsteady Flow Separation over a Circular Cylinder using Dielectric Barrier-Discharge Surface Plasma, Phys. Fluids, 21, 094106 (2009)

[8] Greenblatt, D., Schneider, T., Schüle, C. Y., Mechanism of Flow Separation Control Using Plasma Actuation, Phys. Fluids, 24, 077102 (2012)

[9] Munday, P. M., Taira, K., On the Lock-On of Vortex Shedding to Oscillatory Actuation around a Circular Cylinder, Phys. Fluids, 25, 013601 (2013)

[10] Grundmann, S., Klumpp, S., Tropea, C., Experimental and Numerical Investigations of BoundaryLayer Influence Using Plasma-Actuators, Active Flow Control, Springer, pp. 56-68 (2007)

[11] Hanson, R. E., Lavoie, P., Naguib, A. M., Morrison, J. F., Transient Growth Instability Cancellation by a Plasma Actuator Array, Exp. Fluids, 49, pp. 13391348 (2010)

[12] Choi, K. S., Jukes, T., Whalley, R., Turbulent Boundary-Layer Control with Plasma Actuators, Phil. Trans. R. Soc. A, 369, pp. 1443-1458 (2011)

[13] Kriegseis, J., Schwarz, C., Tropea, C., Grundmann, S. Velocity-Information-Based Force-Term Estimation of Dielectric-Barrier Discharge Plasma Actuators, J. Phys. D: Appl. Phys., 46, 055202 (2013).

[14] Forte. M., Jolibois, J., Pons, J., Moreau, E., Touchard, G., Cazalens, M., Optimization of a Dielectric Barrier Discharge Actuator by Stationary and Non-Stationary Measurements of the Induced Flow Velocity: Application to Airflow Control, Exp. Fluids, 43, pp. 917-928 (2007)

[15] Erfani, R., Erfani, T., Utyuzhnikov, S. V., Kontis, K., Optimization of Multiple Encapsulated Electrode Plasma Actuator, Aerosp. Sci. Technol., 26, pp. 120127 (2013)
[16] Versailles, P., Gringras-Gosselin, V., Vo, H. D., Impact of Pressure and Temperature on the Performance of Plasma Actuators, AIAA J., 48-4, pp. 859863 (2010)

[17] Erfani, R., Zare-Behtash, H., Kontis, K., Plasma Actuator: Influence of Dielectric Surface Temperature, Exp. Therm. Fluid Sci., 42, pp. 258-264 (2012)

[18] Futrzynski, R., Efraimsson, G., Alfredsson, P. H., Numerical Simulation of a Plasma Actuator on a Half-Submerged Cylinder, Submitted to 4th Int. Conf. Jets, Wakes, Sep. Flows, Sept. 17-21, 2013, Nagoya, JAPAN

[19] Likhanskii, A. V., Schneider, M. N., Macheret, S. O., Miles, R. B., Modeling of Dielectric Barrier Discharge Plasma Actuator in Air, J. Appl. Phys., 103, 053305 (2008)

[20] Nishida, H., Abe, T., Numerical Analysis of Plasma Evolution of Dielectric Barrier Discharge Plasma Actuator, J. Appl. Phys., 110, 013302 (2011)

[21] Enloe, C. L., McHarg, M. G., McLaughlin, T. E., Time-Correlated Force Production Measurements of the Dielectric Barrier Discharge Plasma Aerodynamic Actuator, J. Appl. Phys., 103, 073302 (2008)

[22] Neumann, M., Friedrich, C., Czarske, J., Kriegseis, J., Grundmann, S., Determination of the PhaseResolved Body Force Produced by a Dielectric Barrier Discharge Plasma Actuator, J. Phys. D: Appl. Phys., 46, 042001 (2013).

[23] Debien, A., Benard, N., David, L:, Moreau, E., Unsteady Aspect of the Electrohydrodynamic Force Produced by Surface Dielectri Barrier Discharge Actuators, Appl. Phys. Lett., 100, 013901 (2012)

[24] Kotsonis, M., Ghaemi, S., Forcing Mechanisms of Dielectric Barrier Discharge Plasma Actuators at Carrier Frequency of $625 \mathrm{~Hz}$, J. Appl. Phys., 110, 113301 (2011)

[25] Cristofolini, A., Borghi, C. A., Neretti, G., Charge Distribution on the Surface of a Dielectric Barrier Discharge Actuator For The Fluid-Dynamic Control, J. Appl. Phys., 113, 143307 (2013)

[26] Coleman, H. G., Steele, W. G., Experimentation, Validation, and Uncertainty Analysis for Engineers, Third Edition, John Willey \& Sons, Inc., (2009)

\section{Acknowledgments}

The work was financially supported by the Swedish Energy Agency within the project Flow Research on Active and Novel Control Efficiency (FRANCE), project number 341861. Per Elofsson and Guillaume Mercier of Scania $\mathrm{AB}$ are acknowledged for useful input to the project. 